\title{
Blank Holder Force Control System Driven by Servo-Motor
}

\author{
Siji Qin, Li Yang, Bing Yang \\ College of Mechanical Enineering, Yanshan University, Qinhuangdao, China \\ E-mail: plastics@ysu.edu.cn \\ Received September 30, 2011; revised October 28, 2011; accepted November 6, 2011
}

\begin{abstract}
Blank holder force (BHF) control is used to prevent wrinkles of sheet metal in deep drawing process. Based on a novel conception of BHF control technique driven by servo-motor, a new BHF device with six-bar linkage mechanism has been designed and manufactured. Whole control system of the new BHF technique was developed, and the basic structure of the hardware configuration of the system was given. Software analysis, implementation and division of the functional modules have been done. Also, the control software in data acquisition and processing module has been developed in the relevant technology of the BHF control system for the requirements of real-time, stability and accuracy. By the new BHF device combined with the hardware and the software system, the BHF can be regulated accurately variation with the predefined BHF profile in deep drawing process.
\end{abstract}

Keywords: Metal Forming, Blank Holder Force, BHF Control Driven by Servo-Motor, Six-Bar Linkages

\section{Introduction}

In deep drawing, the blank holder plays a role in regulating the metal flow and when the blank holder forces (BHF) selected properly, it can eliminate wrinkles and delay fracture in the drawn part. When the BHF is small, the sheet metal over the flange area tends to instabilities and wrinkling because of excessive circumferential stress, while tensile stress at dangerous section of the sheet metal will increase and fracture occur due to too large BHF. Consequently, the BHF is a key parameter during the process, which should be changed with the punch stroke and an optimal relationship between BHF and stoke for a deep drawing process was called reasonable BHF profile generally [1-3]. The BHF profile can be predicted by numerical simulations, experimental trials and analytical research [1,4-6].

Currently, as a result of large transmission ratio and easy to control, hydraulic transmission is used to implement the variable BHF profile control system [7-9]. Because of their size and complexity, slow response time, control accuracy, and high energy consumption, the hydraulic systems are not suitable for precision, numerical control, flexibility and other requirements in the process of forming.

A new conception for the BHF control driven by a servo motor has been proposed [10]. As many advantages such as short transmission chain, simple control system, numerical control, and so on, this new BHF control system should be more effective to regulate the BHF and to improve the quality of forming parts during the process in comparison with the hydraulic system.

In this paper, according to the BHF control technique based on servo motor driven and the requirements for the BHF in deep drawing process, a whole control system, and its hardware and software have been designed. The designed open-CNC BHF control system is flexible and easy to implement, as well as convenient for further development and application. Also, a BHF actuator consisting of some parts has been designed and manufactured. In the open-CNC technology, the computer is a platform of hardware and software of numerical control system, the motion controller is a key component to control the servo motor and the BHF actuator, so that the blank holder would follow certain movement trajectory, to ensure reasonable BHF profile or other predefined one.

\section{BHF Device Driven by Servo Motor}

The structural principle of blank holder device driven by servo motor is shown in Figure 1. The actuator of the 


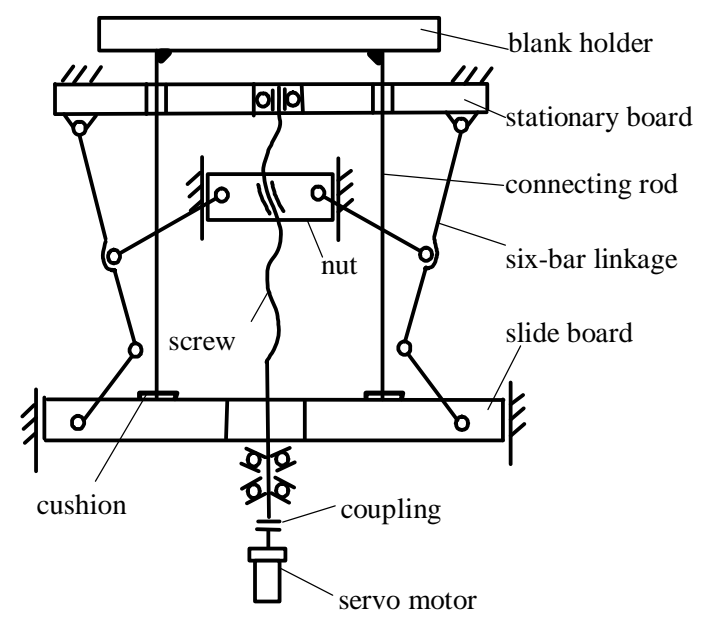

Figure 1. Blank holder device with a six-bar linkage.

BHF control system consists of a ball screw and nut pairs, a six-bar linkage and one blank holder and other parts. In this case, the leadscrew, driven by a servomotor passing through a coupling, is supported between two bearings and housings. The servo motor and the housings are fixed rigidly on a framework. The nut is driven by the ball screw and moves in a straight line. Then, the six-bar linkage driven by the nut passes movement and force to a slide board moving up and down, which is connected to the blank holder by four cushions and four connecting rods. By the six-bar mechanism, the blank holder moves in straight line by variable speed while the nut moves by uniform speed. The relationship between output and input displacement of the six-bar linkage designed is shown in Figure 2, where displacement of the nut and position of the slide board stand for input and output respectively. With the six-bar linkage, the blank holder can be quickly down and slow to load and fast return. In other words, uneven transmission ratio characteristics of the six-bar linkage can greatly reduce the rated motor torque. AC servo motor has a strong overload capacity, wide speed range, good acceleration and deceleration performance, frequent starting, braking, reversing switch and other repetitive motion, so that with a certain power of the AC servo motor and reasonable parameters of the BHF actuator, the blank holder can move by following certain rules and the BHF should vary with the punch stroke to meet the requirements of deep drawing process.

Figure 3 is a BHF device manufactured, which consists of a six-bar linkage, a blank holder, a slide board, four connecting rods, and some other parts.

\section{BHF Control Hardware System}

The implementation and validation of control algorithms requires a flexible structure in terms of hardware and

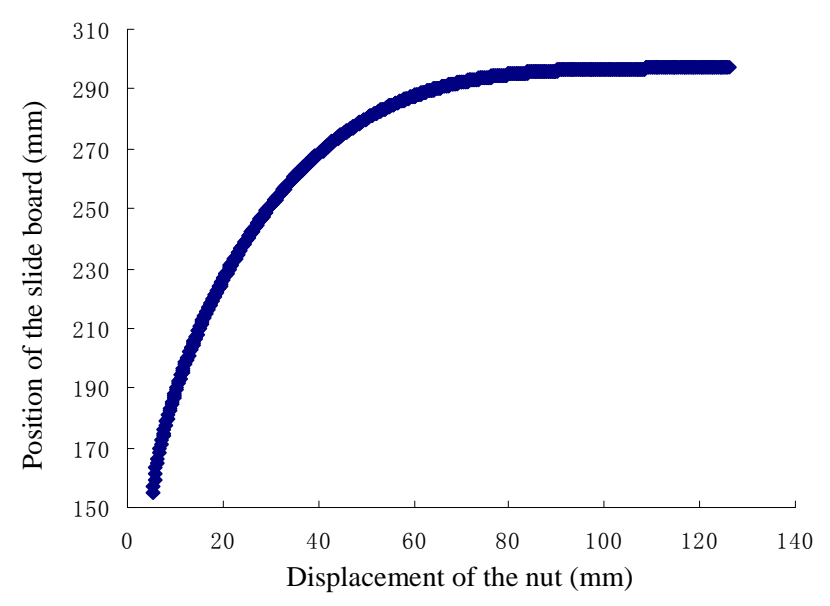

Figure 2. Displacement relationships between input and output for the six-bar linkage.

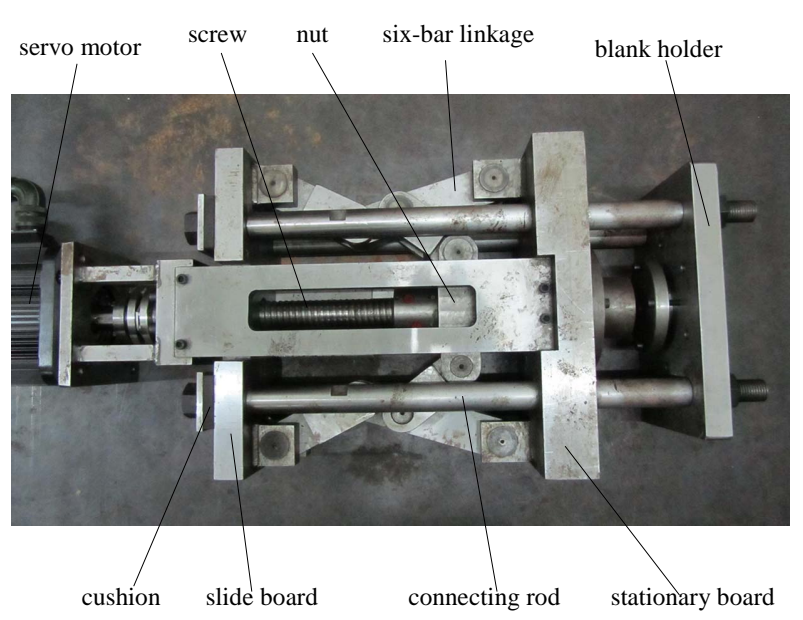

Figure 3. Photo of the blank holder device manufactured.

software. Traditionally, industrial NC systems tasks are generally related to manipulation, which requires only controlling the position of the tools or actuators [11], but in the BHF control, both of the BHF and the position of the blank holder are required.

BHF control system mainly consists of an industrial personal computer (IPC), a motion controller, programmable multi-axis card (PMAC), an AC servo drive and a motor made of Yaskawa, a force sensor, an optical grating, an on-off control, some I/O ports and power units and other components, which is a closed-loop control system (Figure 4).

An IPC-NC hierarchical architecture was used in the BHF system. As an upper computer of the whole system, the IPC is responsible for management, supervision, and control software kernel and background operation.

As a lower computer, the PMAC is a key component of the whole control system, which provides all the realtime instructions for processing and orders the lower 


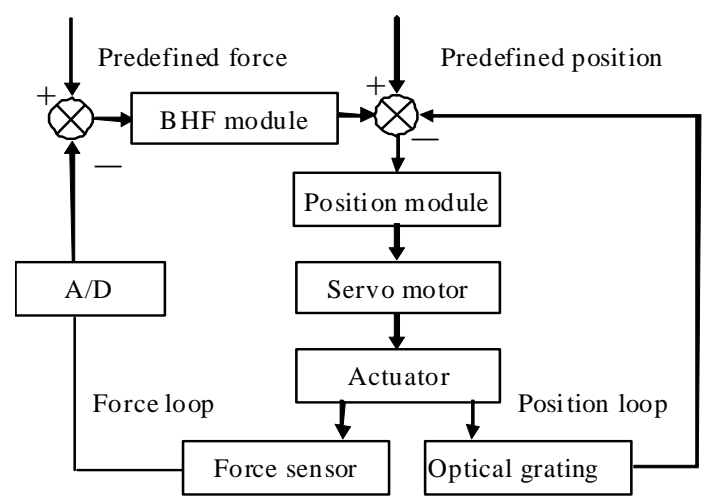

Figure 4. BHF control system block diagram.

control unit simultaneously to cooperate the motion control. The selected PMAC is a 4-axis motion control card, in which, by using high-speed DSP, the CPU has strong programmable logic controlled (PLC) and motion control functions. In the four axes of the PMAC, one is used for the drive of the servo motor and manipulating the BHF actuator, another is for a position control of the press slider. The remaining two can be reserved for development of the system control, one of which may be used for servo feed mechanism in the future.

There are many application examples using PMAC [12-15], some of them involving in force control [14-15]. In the BHF control system, the blank holder position signals feedback from optical grating and access the motion controller, while the BHFs, analog signals, which are acquired by the BHF sensor, are turned into digital signals by A/D converter and processed by filter-amplifier, and then access to the motion controller as feedback signals, and both of the force signals and the position signals are used to realize real-time detection and closedloop control.

\section{BHF Control Software System}

\subsection{BHF Control Strategy}

According to the characteristics of deep drawing process, a composite mode of position control and force control was used. Shown in Figure 5, there are two stages in the whole deep drawing process, one of which is idle stroke, at this time the punch of the press moves up or down fast, and the other is effective work stroke(corresponding to $A B$ segment shown in Figure 5), now the punch moves down slowly. The blank holder should moves rapidly and slowly respectively during the idle stroke and the effecttive work stroke of the punch. When the punch and the blank holder move down, the latter must touch the sheet metal first before the former. During the idle stroke, position manipulating was used in the BHF control system

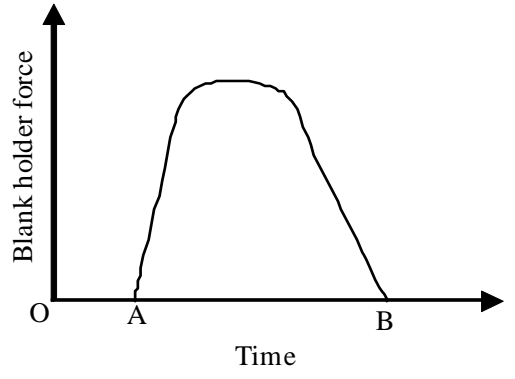

Figure 5. Diagram of BHF versus time.

mainly considering time and efficiency requirements, while during the effective work stroke, blank holder force regulating was used in the BHF system because of the requirements of accurate BHF control.

By programming composition of motion control and directly sending commands to the location of the motion controller, through it the commands are transmitted to the drive and the servo motor, and the blank holder force actuator is driven to achieve the movement of the blank holder, so the position control mode can be realized during idle stroke stage. When the blank holder force sensor detects the signal output is greater than the set value, the control mode is converted from position control to force one. The difference between the BHF detected by the sensor and preset one was input as feedback for closedloop control.

\subsection{Components of the BHF Software System}

BHF control software system consists of non-real-time and real-time control modules. The PC is in charge of the non-real-time motion control, such as non-real-time management, easy operation with the man-machine interface, initialization of the entire BHF system, setting the system parameters, non-real-time display, etc.

For example, a communication module, as non-realtime module, play a role of communication between the PC and the PMAC based on the ethernet technology, by sending online instructions from PComm32 dynamic link library to the PMAC to conduct communication and data exchange to realize the whole control for the system.

Another example of the non-real-time module is human-computer interaction, which has been realized through human-machine interface, and users and the blank holder force control system can exchange information, such as control mode selection, setting of the reasonable BHF profile, speed and location information, displays of the information feedback and working conditions. The users can operate through the software interface, and reliable man-machine interface ensure proper use of system, as well as the data and program security.

Human-machine interface software program was de- 
veloped by $\mathrm{VC}++6.0$ in Windows XP environment. Dynamic link library Pcomm32 was called to realize performances of the motion controller, because API functions in Windows XP have no direct access to the motion controller.

The developed control system main interface is shown in Figure 6.

For example, in the control program for the realization communication between the motion controller and the host computer, the follow codes were written as

CMainFrame::CMainFrame()

\{

hMydll=LoadLibrary(“Pcomm32.dll”); //applied in Pcomm32Pro if(hMydll==NULL)

AfxMessageBox("PMAC Failed to open dll”); open=(OpenPmac)GetProcAddress(hMydll, “OpenPmacDevice”);

$$
\cdots
$$

$$
\}
$$

For real-time control in deep drawing process, the real instantaneous position of the blank holder and the BHF are detected in time and as feedback signals which are compared with predefined values. Then, adjustment signals of the BHF are sent to the drive, so the mechanical actuators can be driven by the servo motor for the closed-loop control.

The performance of the BHF control system depends mainly on the application and development of the software. As a result, it is necessary to develop the BHF control system software considering its own characteristics.

The performance of the real-time control module of the lower computer mainly includes interpolation, position control, BHF acquisition and control, etc. (Figure 7).

The interpolation control and the position control have been encapsulated in the motion control card. This collection focuses on the BHF and its control module. The following focuses on acquisition and control of the BHF.

The following is an example of PLC program for data acquisition:

CLOSE ; Make sure all buffers are closed

DEL ETE GA THER ; Erase any defined gather buffer OPEN PLC10 ; Open buffer for program entry

CL EAR ; Erase existing contents of the buffer

P100 $=0$; Initialization of initial variable

$\mathrm{P} 110=0$; Initialization of BHF

WHILE ( P100 ! < 5) ; To 5 times if not

$\mathrm{P} 110=\mathrm{P} 110+\mathrm{M} 502 ;$ BHF accumulation

ENDWHILE;

$\mathrm{P} 111=\mathrm{P} 110 / 5$; Assignment after averaging

IF ( P111 ! < 2.0) DISABL E PLC10; If the BHF is less than a set value, then close

WHILE ( P10 ! < 10) ; End loop

CLOSE ; Close Buffer

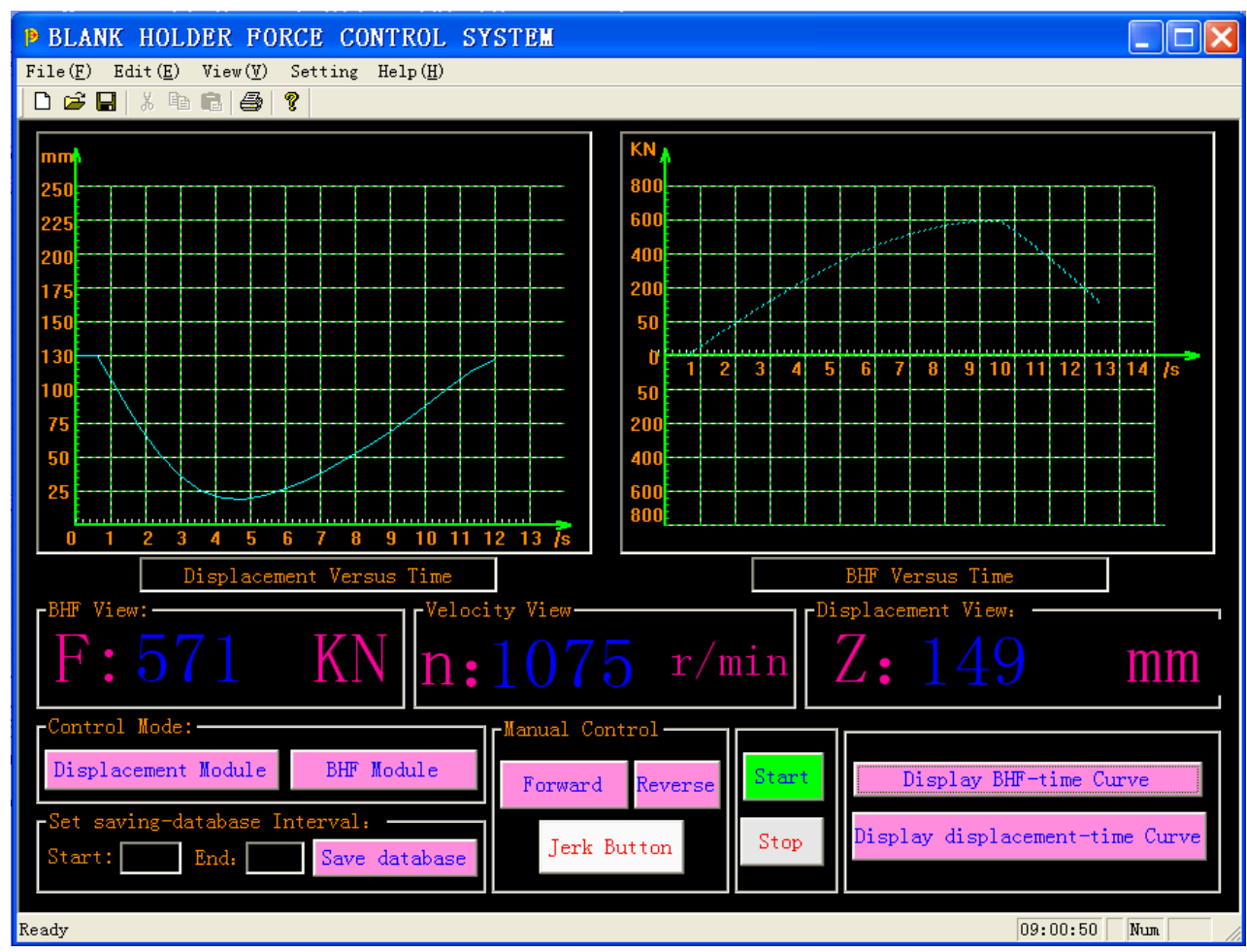

Figure 6. The main interface control system. 


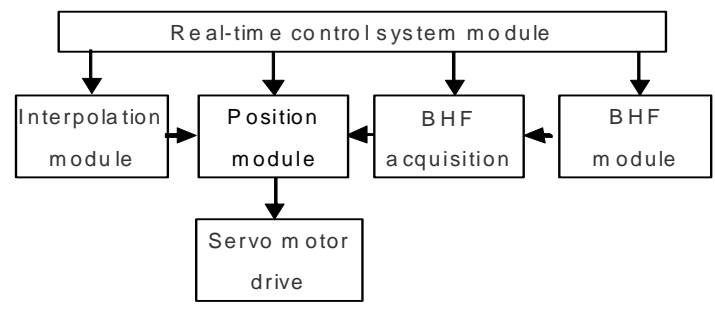

Figure 7. Real-time control system of lower computer.

In the BHF control module, the current feedback BHF value was compared with the predefined one and their difference was converted into pulse. If the BHF value is less than or greater than the current set one, addition or subtraction of the position command signal and several pulses were sent to control the drive and the motor forward or reverse rotates, so the BHF reaches to preset one. The performances of PLC data collection program and the BHF control can be implemented by the PMAC, the control system can achieve real-time response. By timesharing CPU resources of the PMAC, parallel processing can be done according to the priority of the task.

\section{Conclusions}

Based on a novel conception of BHF control technique driven by servo-motor, a new BHF control system has been presented. Some key problems about the system, such as system design and composition, mechanical actuator of the BHF, control strategy and mode, real-time and non-real-time control, and so on, have been investigated. The main results are summarized as follows:

1) A BHF control system driven by servo motor has been designed, which consists of IPC, PMAC, BHF actuator and other hardware components. The blank holder device with a six-bar linkage, a blank holder and other parts has been designed and manufactured.

2) In the BHF system, by using the IPC-NC model, IPC, the host computer for non-real-time control operation and the PMAC, the lower computer for a real-time control, variable blank holder force control can be realized in deep drawing process.

3) According to the characteristics of deep drawing process, a composite mode of position control and force control has been used in the BHF system. The position control and force control were corresponding to the idle stroke and effective work stroke respectively.

4) The BHF software system has been designed by modular method. Many functions of the software system, including non-real-time and real-time modules have been developed. As a result, the proposed system has many advantages such as real-time ability, system stability, control accuracy, easy to operate, and so on, so it can meet the control requirements.

\section{Acknowledgements}

This research was supported by the Hebei Natural Science Foundation (No. 08B014). The authors gratefully acknowledge this support.

\section{References}

[1] Z. Q. Sheng, S. Jirathearanat and T. Altan, "Adaptive FEM Simulation for Prediction of Variable Blank Holder Force in Conical Cup Drawing," International Journal of Machine Tools \& Manufacture, Vol. 44, No. 5, 2004, pp. 487-494. doi.10.1016/j.ijmachtools.2003.11.001

[2] E. J. Obermeyer and S. A. Majlessi, “A Review of Recent Advances in the Application of Blank Holder Force towards Improving the Forming Limits of Sheet Metal Parts," Journal of Materials Processing Technology, Vol. 75, No. 1-3, 1998, pp. 222-234. doi.10.1016/S0924-0136(97)00368-3

[3] K. Siegert, E. Dannenmann, S. Wagner and A. Galeiko, "Closed-Loop Control System for Blank Holder Forces in Deep Drawing,” Annals of the CIRP, Vol. 44, No. 1,1995, pp. 251-254.

[4] D. M. Rodrigues, C. Leitao and L. F. Menezes, “A Multi-Step Analysis for Determining Admissible BlankHolder Forces in Deep-Drawing Operations,” Materials and Design, Vol. 31, No. 3, 2010, pp. 1475-1481. doi.10.1016/j.matdes.2009.08.028

[5] W. Thomas, "Product Tool and Process Design Methodology for Deep Drawing and Stamping of Sheet Metal Parts," PhD Thesis, Ohio State University, Columbus, 1999.

[6] D. E. Hardt and R. C. Fenn, "Real-Time Control of Sheet Stability during Forming," ASME Journal of Engineering for Industry, Vol. 115, No. 3, 1993, pp. 299-308.

[7] T. Yagami, K. Manabe and Y. Yamauchi, "Effect of Alternating Blank Holder Motion of Drawing and Wrinkle Elimination on Deep-Drawability," Journal of Materials Processing Technology, Vol. 187-188, 2007, pp. 187-191.

[8] H. B. Sim and M. C. Boyce, "Finite Element Analyses of Real-Time Stability Control in Sheet Forming Processes," Journal of Materials Processing Technology, Vol. 114, No. 1, 1992, pp. 180-188

[9] J. Zhao, H. Q. Cao, L. X. Ma, et al., "Study on Intelligent Control Technology for the Deep Drawing of an AxiSymmetric Shell Part,” Journal of Materials Processing Technology, Vol. 151, No. 1-3, 2004, pp. 98-104. doi.10.1016/j.jmatprotec.2004.04.023

[10] S. J. Qin, “State-of-the-Art of Blank Holding Force Control Technology and Feasibility of Numerical Servo-Control Holding,” China Mechanical Engineering, Vol. 18, No. 1, 2007, pp. 120-125.

[11] L. Liu, Y. Li, L. W. Wen and J. Xiao, "PMAC-Based Tracking Control System for 8-Axis Automated TapeLaying Machine,” Chinese Journal of Aeronautics, Vol. 22, No. 5, 2009, pp. 559-563. doi.10.1016/S1000-9361(08)60141-7 
[12] K.-S. Honga, K.-H. Choib, J.-G. Kimc and S. Lee, “A PC-Based Open Robot Control System: PC-ORC,” Robotics and Computer Integrated Manufacturing, Vol. 17, No. 4, 2001, pp. 355-365. doi.10.1016/S0736-5845(01)00010-2

[13] I. Kim, N. Nakazawa, S. Kim, C. Park and C. Yu, “Compensation of Torque Ripple in High Performance BLDC Motor Drives," Control Engineering Practice, Vol. 18, No. 10, 2010, pp. 1166-1172. doi.10.1016/j.conengprac.2010.06.003

[14] P. Charbonnaud, F. J. Carrillo and D. Ladevèze, "Monitored Robust Force Control of a Milling Process," Control Engineering Practice, Vol. 9, No. 10, 2001, pp. 1047-1061.doi.10.1016/S0967-0661(01)00074-0

[15] Y. C. Jiang and F. J. Ren, "Constant Tension Control System of Wire Tool in WEDM-HS Based on PMAC," China Mechanical Engineering, Vol. 19, No. 16, 2007, pp. 1047-1061. 